\title{
Mixed Systems of Hydrophobically Modified Polyelectrolytes: Controlling Rheology by Charge and Hydrophobe Stoichiometry and Interaction Strength ${ }^{\dagger}$
}

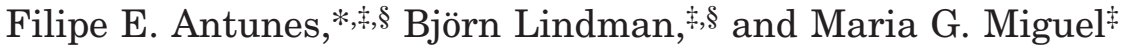 \\ Department of Chemistry, University of Coimbra, 3004-535 Coimbra, Portugal, and Physical \\ Chemistry 1, Center for Chemistry and Chemical Engineering, Lund University, P.O. Box 124, \\ 22100 Lund, Sweden
}

Received March 4, 2005. In Final Form: April 14, 2005

\begin{abstract}
Rheology and phase separation were investigated for aqueous mixtures of two oppositely charged hydrophobically modified polyelectrolytes. The typical phase separation, normally seen for oppositely charged polymer mixtures, is dramatically reduced by the presence of hydrophobic modification, and phase separation is only detected close to the point of charge neutralization. While the two polyelectrolytes separately can give high viscosities and a gel-like behavior, a pronounced maximum in viscosity and storage modulus with the mixing ratio of the polyelectrolytes is observed; the maximum is located between the points of charge and hydrophobe stoichiometry and reflects a combination of hydrophobic and electrostatic association. Lowering the charge density of the anionic polymer leads to a strengthened association at first, but at lower charge densities there is a weakened association due to the onset of phase separation. The strength of the electrostatic interaction was modified by adding salt. Increased ionic strength can lead to phase separation and to increased or decreased viscosity depending on the polyelectrolyte mixing ratio.
\end{abstract}

\section{Introduction}

Hydrophobically modified water-soluble polymers give, because of intermolecular hydrophobic association, rise to considerable increases in viscosity. This has led to several applications for rheology control of formulations ranging from paints and personal care products to pharmaceuticals. ${ }^{1-6}$ The viscosity increase depends, in addition to molecular mass, strongly on hydrophobe size and density but also on whether the polymer is ionic or nonionic.

Formulations where these polymers are used typically also contain one or more surfactants. The interaction between the hydrophobically modified polymer (HMP) and the surfactant(s) typically has very strong effects on rheology, with both increases and decreases possible, mainly depending on the surfactant-polymer hydrophobe stoichiometry and surfactant-polymer charge relation.

An understanding of these features is based on our understanding of polymer-surfactant interactions in general and polymer-induced surfactant self-assembly and phase separation phenomena in particular. A general effect is that ionic surfactant self-assembly is facilitated by the presence of a polymer, leading to a decreased critical

* Corresponding author: Department of Chemistry, University of Coimbra, 3004-535 Coimbra, Portugal; e-mail, fcea@ci.uc.pt; phone, +351 239 852080; fax, +351 239827703.

$\dagger$ Part of the Bob Rowell Festschrift special issue.

$¥$ University of Coimbra.

$\S$ Lund University.

(1) Tanford, C. The Hydrophobic Effect: Formation of Micelles and Biological Membranes, 2nd ed.; Wiley: New York, 1980.

(2) Glass, J. E. Polymers in Aqueous Media; American Chemical Society: Washington, DC, 1989; Vol. 223.

(3) Karlson, L. Hydrophobically Modified Polymers. Rheology and Molecular Associations. Ph.D. Thesis, Lund University, 2002

(4) Karlson, L.; Joabsson, F.; Thuresson, K. Carbohydr. Polym. 2000, $41,25$.

(5) Inoue, T.; Chen, G.; Nakamae, K.; Hoffman, A. J. Controlled Release 1997, 49, 167.

(6) Antunes, F. E.; Thuresson, K.; Lindman, B.; Miguel, M. G. Colloids Surf., A 2003, 215, 87 . micelle concentration, cmc. The effect is small for more hydrophilic homopolymers but becomes more pronounced for less polar, slightly hydrophobic polymers. For the case of oppositely charged surfactant-polymer pairs, the effect becomes very large and the cmc may be reduced by orders of magnitude. A common feature of these oppositely charged mixtures is an associative phase separation, leading to a concentrated phase of stoichiometric amounts of polymer and surfactant, in equilibrium with a dilute phase containing any excess of one of the cosolutes. ${ }^{7-9}$

With HMPs, the interaction with surfactant is quite different and has different consequences. While the surfactant binding to homopolymers is typically strongly cooperative, the binding isotherm is more complex with HMPs. While at low surfactant concentrations, there is a low amount of noncooperative binding, there is then an anticooperative plateau before the typically cooperative surfactant binding. The effects on rheology of surfactant binding are typically dramatic, and the viscosity may increase by orders of magnitude on addition of quite small amounts of surfactant to a solution of an HMP. However, for higher surfactant concentrations, there is typically a major decrease of viscosity, reaching even levels much below the surfactant-free case. ${ }^{10-12}$ These effects are now well understood in terms of cross-link lifetimes and micelle-polymer hydrophobe stoichiometry. The viscosity increase for the polymer alone is due to formation of hydrophobic cross-links or hydrophobic microdomains associating different polymer chains creating a threedimensional network. Surfactant binding to these aggregates leads to larger aggregates and thus to an

(7) Khokhlov, A. R.; Kramarenko, E. Y.; Makhaeva, E. E.; Starodubtzev, S. G. Macromolecules 1992, 25, 4779.

(8) McQuigg, D. W.; Kaplan, J. I.; Dubin, P. L. J. Phys. Chem. 1992 96, 1973.

(9) Wang, C.; Tam, K. C. J. Phys. Chem. B 2004, 108, 8976.

(10) Tanaka, R.; Meadows, J.; Williams, P. A.; Phillips, G. O. Macromolecules 1992, 25, 1304.

(11) Iliopoulos, I.; Wang, T. K.; Audebert, R. Langmuir 1991, 7, 617

(12) Nilsson, S.; Thuresson, K.; Lindman, B.; Nystrom, B. Macromolecules 2000, 33, 9641 . 
increased micelle lifetime, in turn giving a higher viscosity. However, for a surfactant forming spherical or other micelles of limited size, there will be a limit for the viscosity increase when the micelle number approaches the number of polymer hydrophobes; after that the number of crosslinks decreases with surfactant binding and there will ultimately be a complete decoupling of polymer chains.

The viscosity increases are especially large for oppositely charged HMP-surfactant mixtures, because of the combined role of hydrophobic and electrostatic associations. ${ }^{13-16}$ However, these mixtures share the general feature of associative phase separation with polyelectrolyte-oppositely charged surfactant mixtures in general. The range of phase separation becomes, however, strongly reduced as the polyelectrolyte is hydrophobically modified. As explained by Piculell ${ }^{17}$ this can be referred to the stoichiometry of the formed polymer-surfactant complexes. For polyelectrolytes in general, the situation of a stoichiometric polyion-ionic surfactant complex in one phase in equilibrium with excess polyelectrolyte in a separate phase is a stable one. For a hydrophobically modified polyelectrolyte, the hydrophobic association leads to a strong tendency to form also nonstoichiometric complexes, i.e., complexes with a net charge. Because of the counterions, the entropic driving force for mixing of these complexes is large. Therefore, hydrophobic modification of the polyelectrolyte strongly reduces the tendency for phase separation, which is only observed in a quite narrow range around charge stoichiometry.

A natural extension of these studies of HMP-surfactant mixtures, in particular as a way to developing novel, more efficient, and more robust thickening and gelling systems, was to consider mixtures of two oppositely charged HMPs. It was indeed found that dramatic increases in viscosityup to 4 orders of magnitude-were obtained relative to the separate HMPs, when used individually as thickeners. It was also observed that the associative phase separation between the polymers-very extensive for mixed polyelectrolytes in general ${ }^{18-21-}$ was very strongly inhibited and occurred only in a very narrow range around charge stoichiometry. The explanation was argued to be analogous to that mentioned above for mixed systems of a polyelectrolyte and an oppositely charged surfactant.

To both increase our understanding and improve our ability to design efficient thickening systems and gels, it was deemed important not only to study the effect of the mixing ratio of the polyelectrolytes but also to investigate the effect of charge and ion stoichiometry as well as the strength of interaction; the latter can be modified both by changing the hydrophobicity of the modification and by varying the ionic strength. The present investigation is a step in this direction. We have here investigated aqueous mixtures of two cellulose derivates, both hydrophobically

(13) Bai, G. Y.; Santos, L.; Nichifor, M.; Lopes, A.; Bastos, M. J. Phys. Chem. B 2004, 108, 405

(14) Nichifor, M.; Zhu, X.; Cristea, D.; Carpov, A. J. Phys. Chem. B $\mathbf{2 0 0 1}, 105,2314$

(15) Antunes, F. E.; Marques, E. F.; Gomes, R.; Thuresson, K.; Lindman, B.; Miguel, M. G. Langmuir 2004, 20, 4647.

(16) Magny, B.; Iliopoulos, I.; Zana, R.; Audebert, R. Langmuir 1994, 10,3180 .

(17) Piculell, L.; Guillemet, F.; Thuresson, K.; Shubin, V.; Ericsson, O. Adv. Colloid Interface Sci. 1996, 63, 1 .

(18) Thuresson, K.; Nilsson, S.; Lindman, B. Langmuir 1996, 12 530.

(19) Tsianou, M.; Kjoniksen, A. L.; Thuresson, K.; Nystrom, B. Macromolecules 1999, 32, 2974.

(20) Holmberg, K.; Jonsson, B.; Kronberg, B.; Lindman, B. Surfactants and Polymers in Aqueous Solution, 2nd ed.; John Wiley Sons: New York, 2003.

(21) Evans, D.; Wennerstrom, H. The Colloidal Domain: Where Physics, Chemistry, and Biology Meet; Wiley-VCH: New York, 1999. modified: one cationic with quaternary ammonium groups and one anionic with carboxylate groups. The charge density of the polyanion can be varied by changing the $\mathrm{pH}$. Furthermore, we modified the strength of interaction between the polyions by varying the ionic strength. Our investigation has been focused on rheological effects but included as a natural part phase separation phenomena.

The polymers chosen for study both have a cellulose backbone but show otherwise important differences: the cationic $\left(\mathrm{HMP}^{+}\right)$has a lower charge density but a higher hydrophobe density than the anionic one $\left(\mathrm{HMP}^{-}\right)$; the cationic has a charge density, which is independent of $\mathrm{pH}$, while the charge density of the anionic can be varied by changing the $\mathrm{pH}$; the anionic is more water soluble while the cationic is easily salted out.

\section{Experimental Section}

Materials. Hydrophobically modified carboxymethyl cellulose $\left(\mathrm{HMCMC}\right.$ here denoted $\mathrm{HMP}^{-}$) is a semiflexible polyanion, ${ }^{22}$ in the sodium salt form; it was supplied by Akzo Nobel Surface Chemistry. CMC is modified by tetradecyl groups with a degree of hydrophobic substitution of $1.2 \%$ ( $1.2 \mathrm{C}_{14}$ chains per 100 sugar units) and a charge density (percentage of carboxymethyl groups) of $80 \%$ at $\mathrm{pH} 8$ ( 80 negative charges per 100 sugar units). The weight average molecular weight is around $879000 \mathrm{~g} / \mathrm{mol}$ and the number average is 289000 ; thus the polydispersity is around 3. From the charge density it can be deduced that a $1 \mathrm{wt} \%$ solution has a concentration of charges which is $33.6 \mathrm{mM}$; the overlap concentration is around $0.06 \mathrm{wt} \% .{ }^{23} \mathrm{CMC}$ is fully ionized at $\mathrm{pH} 7$ and is almost nonionic at $\mathrm{pH} 2 .{ }^{22,24}$

The cationic polymer, Quatrisoft LM200 $\left(\mathrm{HMP}^{+}\right)$is a semiflexible polycation provided by Union Carbide Chemicals and Plastics Company, Inc., as the chloride salt of a $N, N$-dimethyl$\mathrm{N}$-dodecyl derivative of hydroxyethyl cellulose with a molar mass of ca. $100000 \mathrm{~g} / \mathrm{mol} \cdot{ }^{25} \mathrm{LM} 200$ has a degree of dodecyl substitution of $5.4 \%$ ( $5.4 \mathrm{C}_{12}$ per 100 sugar units) and a charge density of $5.4 \%$ (5.4 positive charges per 100 sugar units). ${ }^{26}$ Its overlap concentration is around $2 \mathrm{~g} / \mathrm{dL} .{ }^{23}$ The charges are located at the hydrophobic tails. A $1 \mathrm{wt} \%$ aqueous solution has a charge concentration of $2 \mathrm{mM} .{ }^{15}$ Thus, $\mathrm{HMP}^{-}$has a much higher charge density than $\mathrm{HMP}^{+}$. By comparison of the charge densities, it can be deduced that charge neutrality occurs when the weight fraction of $\mathrm{HMP}^{-}$is 0.056 . From the degrees of hydrophobic modification, it can be deduced that the hydrophobe stoichiometry occurs at a weight fraction of $\mathrm{HMP}^{-}$which is 0.80 .

Polymers and salt $(\mathrm{NaCl})$ were used without further purification.

Methods. The samples were prepared by weight using appropriate amounts of the two solid polymers. Pure Millipore water was used. After around $3 \mathrm{~min}$ in a vortex mixer, samples were centrifuged at $3200 \mathrm{~g}$ for around $4 \mathrm{~h}$. The cycle was repeated around five times. The last centrifuging process was performed around $12 \mathrm{~h}$ since it was observed that HMCMC gel mixtures produce foam. Liquid samples were placed in a platform stirrer for $48 \mathrm{~h}$. During sample preparation, energy input from mechanical deformations, as centrifuging and vortex or heating, does not influence the rheological response. This was investigated by comparing rheological information of two samples $(0.5 \mathrm{wt} \%$ HMCMC) prepared in different ways: one was submitted to standard preparation method and the other was submitted to gentle vortex mixing, gentle centrifuging, and 1 week in the stirrer platform. The samples show very similar storage responses (1.4 and 1.6 $\mathrm{Pa}$ in standard and gentle preparations, respectively). Biphasic regions in the phase map were identified by the following criterion: when the top and bottom phases coexist in sample

(22) Hoogendam, C. W.; Keizer, A.; Cohen Stuart, M. A.; Bijsterbosh, B. H. Macromolecules 1998, 31, 6297.

(23) Alvarez-Lorenzo, C.; Duro, R.; Goméz-Amoza, J. L.; MartínezPacheco, R.; Souto, C.; Concheiro, A. Colloid Polym. Sci. 2001, 279, 1045 .

(24) Cohen Stuart, M. A.; Fokkink, R. G.; van der Horst, P. M.; Lichtenbelt, J. W. Th. Colloid Polym. Sci. 1998, 276, 335.

(25) Goddard, E. D.; Leung, P. S. Colloids Surf. 1992, 65, 211.

(26) Guillemet, F.; Piculell, L. J. Phys. Chem. 1995, 99, 9201. 


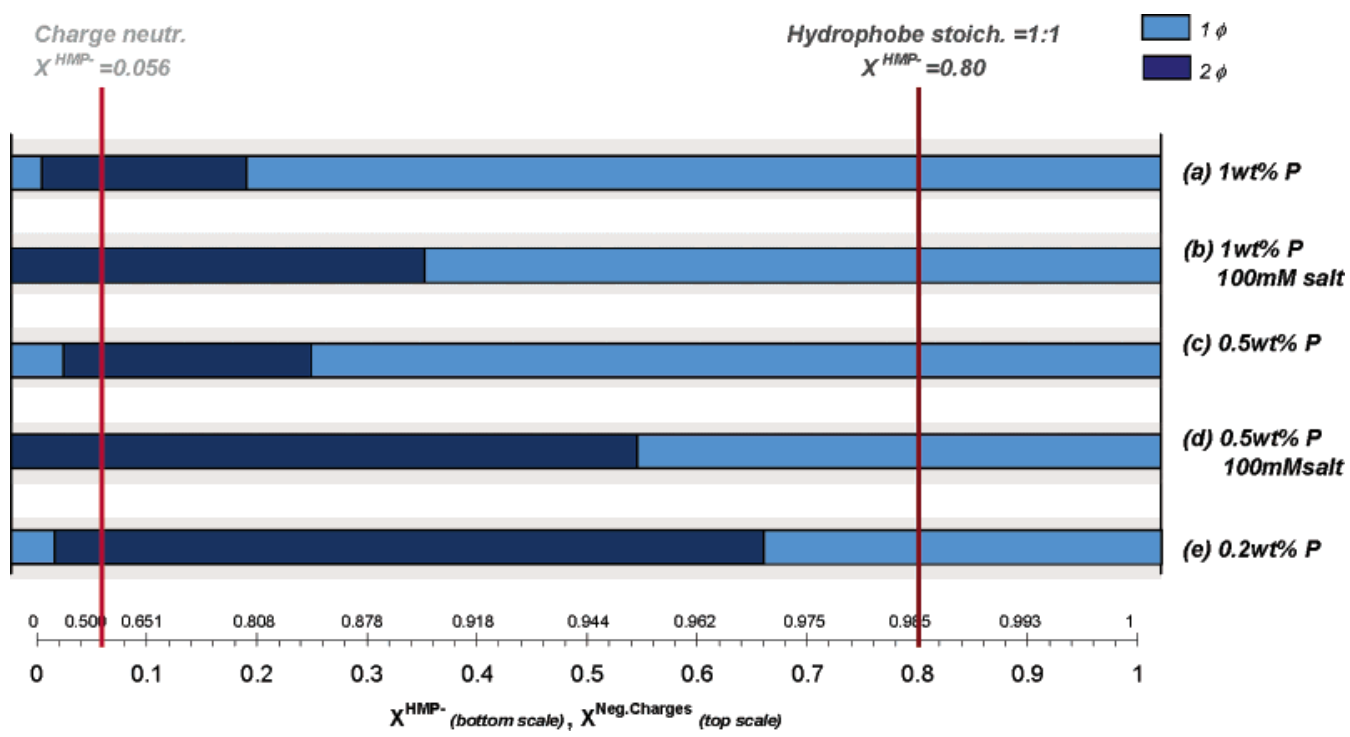

Figure 1. Phase map of $\mathrm{HMP}^{+} \mathrm{HMP}^{-}$mixture at different weight and charge fractions. Monophasic regions are represented by a light gray area while biphasic regions are indicated by a black area. Map a: 1 wt \% total polymer concentration, salt-free. Map b: 1 wt \% total polymer concentration, $100 \mathrm{mM}$ salt. Map c: $0.5 \mathrm{wt} \%$ total polymer concentration, salt-free. Map d: $0.5 \mathrm{wt} \%$ total polymer concentration, $100 \mathrm{mM}$ salt. Map e: $0.2 \mathrm{wt} \%$ total polymer concentration, salt-free.

tube after centrifugation at $3200 \mathrm{~g}$. Monophasic samples were reanalyzed several weeks after the preparation and no phase separation was detected again, which is an indication of equilibrium.

A Reologica StressTech rheometer with automatic gap setting was used. Frequency sweep measurements were performed in the linear viscoelastic region (where $G^{\prime}$ and $G^{\prime \prime}$ are independent of the applied stress) and all rotational flow curves were obtained using steady state. An acrylic cone with $50 \mathrm{~mm}$ of diameter and $1^{\circ}$ was used. All rheological measurements were performed at $25.0{ }^{\circ} \mathrm{C}$.

Stock solutions of $0.5,0.1$, and $0.01 \mathrm{M}$ of $\mathrm{HNO}_{3}$ and $\mathrm{NaOH}$ were prepared for $\mathrm{pH}$ studies. A micro-pH glass electrode was used for $\mathrm{pH}$ measurements.

\section{Results and Discussion}

Phase Separation. Mixtures of $\mathrm{HMP}^{+}$and $\mathrm{HMP}^{-}$were investigated with respect to stability and phase separation at different mixing ratios at total polymer weight percentages of $0.2,0.5$, and 1 , without salt and in the presence of $100 \mathrm{mM} \mathrm{NaCl}$. For $\mathrm{HMP}^{+}, C / C^{*}\left(C^{*}\right.$ being the overlap concentration) is around $1.0,2.5$, and 5.0 (for $0.2,0.5$, and 1.0 wt $\%$, respectively). For $\mathrm{HMP}^{-}, C / C^{*}$ is $3.3,8.3$, and 16.7 (for $0.2,0.5$, and $1.0 \mathrm{wt} \%$, respectively). The experimental findings are summarized in Figure 1.

Let us first consider the solubility of the individual polymers. Polyelectrolytes are typically soluble in water. In many cases the solubility is dependent on the mixing entropy of the dissociating counterions; in other cases the polyelectrolytes are intrinsically soluble. For the present polymers we noted that even a relatively low concentration of salt $(20-50 \mathrm{mM})$ is sufficient to induce phase separation in the case of $\mathrm{HMP}^{+}$; this "salting-out" has already been noted in the literature. ${ }^{26}$ Other polyelectrolytes owing their solubility to the counterion entropy include poly(styrene sulfonate) and many proteins (insoluble at the isoelectric point). $\mathrm{HMP}^{-}$appears to be closer to intrinsically soluble polyelectrolytes, like for example dextran sulfate and polyacrylate, since high concentrations of salt (1 M) do not cause phase separation in a $1 \mathrm{wt} \%$ polymer solution. Only with $1 \mathrm{M}$ of salt in a $0.2 \mathrm{wt} \% \mathrm{HMP}^{-}$solution is a phase separation observed. (On the other hand, lowering the $\mathrm{pH}$ to ca. $\mathrm{pH} 3.2$ leads to reduced solubility-less than $1 \mathrm{wt} \%$.) This difference in solubility behavior between the two polyelectrolytes is a key to understanding not only the phase separation in mixed polymer solutions but also some of the rheological effects. We also note that the phase separation of $\mathrm{HMP}^{-}$, occurring at high salt, is more significant for a low polymer concentration. This behavior is similar to that observed for hydrophobically modified nonionic polymers (which can be strongly dependent on temperature) and is due to the strong hydrophobic coupling between the "stickers" on the polymer molecules. Phase separation induced on dilution is indeed well-known for simple or complex polymer solutions if there is a strong association between polymer chains. A rather concentrated system will be one phase, while on dilution it will swell to a certain water content until the polymer chains are stretched. At this point, a phase separation will occur and a water-rich phase will form. (Upon further dilution a one-phase region is again entered since the physical attraction is not strong enough to conserve the initial structure. Smaller clusters will be found in the solution instead of the macroscopic physical gel.)

The two oppositely charged polyelectrolytes, $\mathrm{HMP}^{+}$and $\mathrm{HMP}^{-}$, have different charge densities and hydrophobic modifications, as described above. Charge neutrality occurs at a weight fraction $X_{\mathrm{HMP}^{-}}$of 0.056 , i.e., in the $\mathrm{HMP}^{+}$ dominating region. As mentioned, Figure 1 indicates the phase diagram of the mixtures at different conditions. The total polymer concentration used was $1,0.5$, and 0.2 wt $\%$. The $\mathrm{HMP}^{+}$concentration varies from 0 to $0.100 \mathrm{mM}$ ( 1 wt \%) while $\mathrm{HMP}^{-}$is present between 0 and $0.0125 \mathrm{mM}$ (1 wt \%). Considering first the $1 \mathrm{wt} \%$ salt-free situation (a), a monophasic region is detected at almost all mixing ratios. The samples are transparent with a gel-like consistency. Only around $X_{\mathrm{HMP}^{-}}=0.056$, phase separation is obtained, with a gel-like bottom phase (occupying from 10 to $70 \% \mathrm{v} / \mathrm{v}$ ) in equilibrium with a transparent low viscous top phase. Since this polymer ratio corresponds to a situation where positive and negative charges from the two polymers match (charge stoichiometry 1:1), this can be identified as an associative phase separation (sometimes in the literature denoted "complex coacervation"). This associative phase separation in mixed polyelectrolyte systems is entropically driven: Highly charged polyions attract a large number of counterions; on association between oppositely charged pairs these are 
liberated and entropy increases. Here we observe the expected phase separation but in a much narrower range of mixing ratios than for polyelectrolytes in general. This we ascribe to the presence of not only attractive electrostatic interactions but also attractive hydrophobic interactions between polyions. Without hydrophobic modification, the situation with a stoichiometric concentrated phase and an excess polyelectrolyte solution is a stable situation, leading to phase separation over a wide range of mixing ratios. However, when both polyelectrolytes are hydrophobically modified, there is a large tendency to form nonstoichiometric complexes; i.e., complexes with a net charge, which because of their polyion nature have a high aqueous solubility. Analogous observations were made previously for other oppositely charged HMP systems. ${ }^{18,19,27}$

While the narrow range of phase separation is thus readily understood, it is less trivial to interpret the increased range over which phase separation takes place as the total polymer concentration is reduced (to 0.5 and $0.2 \mathrm{wt} \%$ ). However, it appears that we can attribute these findings to a similar mechanism as discussed above in conjunction with the phase separation in solutions of a single polymer. Thus, due to attractive hydrophobic and electrostatic interactions between the polymer chains, a three-dimensional network is formed. For a high enough polymer concentration a homogeneous solution results. However, as the solution is diluted the polymer chains are stretched leading to a loss of conformational entropy. At a certain concentration, phase separation into one concentrated and one dilute solution becomes more stable. Therefore, the increased phase separation on dilution from 0.5 to $0.2 \mathrm{wt} \%$ is suggested to be due to a strong attractive interaction between the polymer chains.

Addition of salt leads to a considerably broadened range of phase separation. This is in line with expectation, as the nonstoichiometric polymer complexes would own their solubility to the mixing entropy of dissociating counterions; on salt addition this driving force will be reduced. In line with the interpretation given, the phase separation region is largest for the $0.5 \mathrm{wt} \%$ polymer mixture in the presence of salt, occupying around $40 \%$ of the phase map.

Rheology. Varying the Stoichiometry of Mixtures of $\mathbf{H M P}^{+}$and $\mathbf{H M P}^{-}$. The rheological properties of aqueous mixtures composed of the oppositely charged hydrophobically modified polyelectrolytes were investigated in different ways. The study included determining the storage and loss moduli and the complex viscosity as a function of frequency in frequency sweep measurements and the shear viscosity as a function of stress. The study concerned not only varying the charge and hydrophobe ratios but also modifing the electrostatic association by adding salt. We will first discuss the results pertaining to different mixing ratios of the polymers in the absence of salt and come back to the effect of adding salt and varying the $\mathrm{pH}$ below.

To add to the understanding of the nature of the phase separations detected, we not only examined the homogeneous mixtures but also analyzed the transparent top phases of selected samples by rheology. Table 1 presents the relative viscosities of several samples. The results confirm clearly the notion of an associative phase separation. For example, in the sample with a value $X_{\mathrm{HMP}^{-}}$of 0.1 , the bottom phase (a strong bluish gel) occupies 10-15\% (v/v). The top phase has a viscosity similar to that of water, which can be understood as a dilute phase.

(27) Nyrkova, I. A.; Khokhlov, A. R.; Doi, M. Macromolecules 1994 27,4220 .
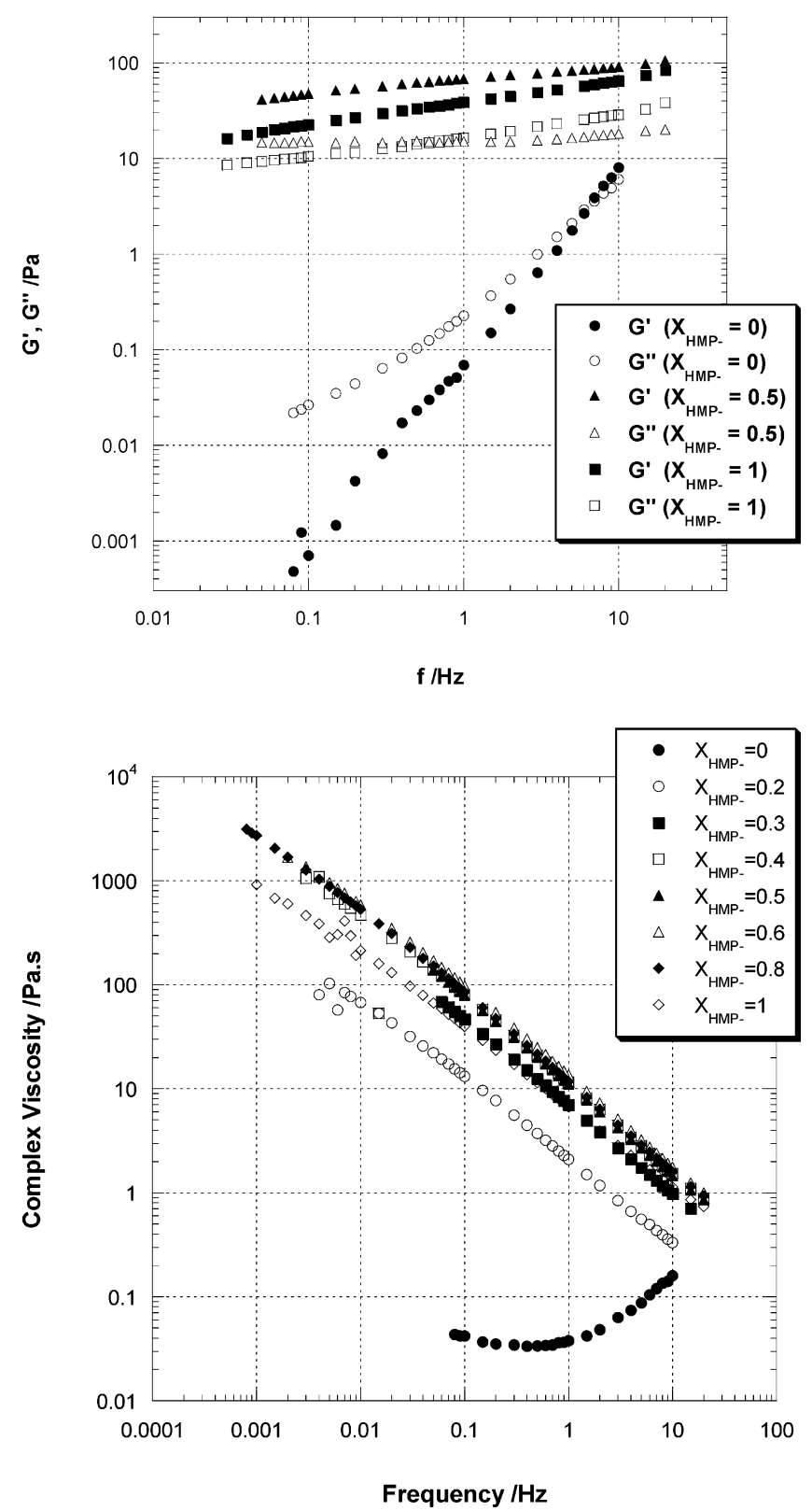

Figure 2. (a) Frequency dependence of the storage modulus $\left(G^{\prime}\right)$ and loss modulus $\left(G^{\prime \prime}\right)$ for polymer mixtures of different weight fractions at a total polymer concentration of $1 \mathrm{wt} \%$. (b) Dependence of complex viscosity on frequency for polymer mixtures with different weight fractions at a total polymer concentration of $1 \mathrm{wt} \%$.

Table 1. Shear Viscosity $(\eta)$ and Relative Viscosity $\left(\eta_{\text {rel }}\right)$ for Some Top Phase Samples at Different $X_{\mathrm{HMP}^{-}}$and at a Total Polymer Concentration of 0.5 and 1 wt \%, with and without Salt

\begin{tabular}{|c|c|c|c|}
\hline top phase sample & $\begin{array}{c}\% \text { top } \\
\text { phase v/v }\end{array}$ & $\begin{array}{c}\eta^{* / \mathrm{Pa} \cdot \mathrm{s}} \\
(\sigma=0.01 \mathrm{~Pa})\end{array}$ & $\begin{array}{c}\eta_{\text {rel }} \\
\left(=\eta / \eta_{\text {water }}\right)\end{array}$ \\
\hline 1 wt $\%, X_{\mathrm{HMP}^{-}}=0.1$ & 90 & $1.52 \times 10^{-3}$ & 1.52 \\
\hline $1 \mathrm{wt} \%, X_{\mathrm{HMP}^{-}}=0.12$ & 80 & $1.05 \times 10^{-3}$ & 1.05 \\
\hline 1 wt $\%, X_{\mathrm{HMP}^{-}}=0.12$ & 70 & $1.16 \times 10^{-3}$ & 1.16 \\
\hline 1 wt $\%, X_{\mathrm{HMP}^{-}}=0.16$ & 60 & $1.75 \times 10^{-3}$ & 1.75 \\
\hline $\begin{array}{c}1 \text { wt } \%, X_{\mathrm{HMP}^{-}}=0.13, \\
100 \mathrm{mM} \text { salt }\end{array}$ & 40 & $3.16 \times 10^{-3}$ & 3.16 \\
\hline 0.5 wt $\%, X_{\mathrm{HMP}^{-}}=0.5$ & 40 & $3.11 \times 10^{-3}$ & 3.11 \\
\hline
\end{tabular}

$100 \mathrm{mM}$ salt

Figures 2-4 show different rheological results for a total polymer concentration of $1 \mathrm{wt} \%$. Figure $2 \mathrm{a}$ shows the dependence of viscoelasticity on frequency. While 18 


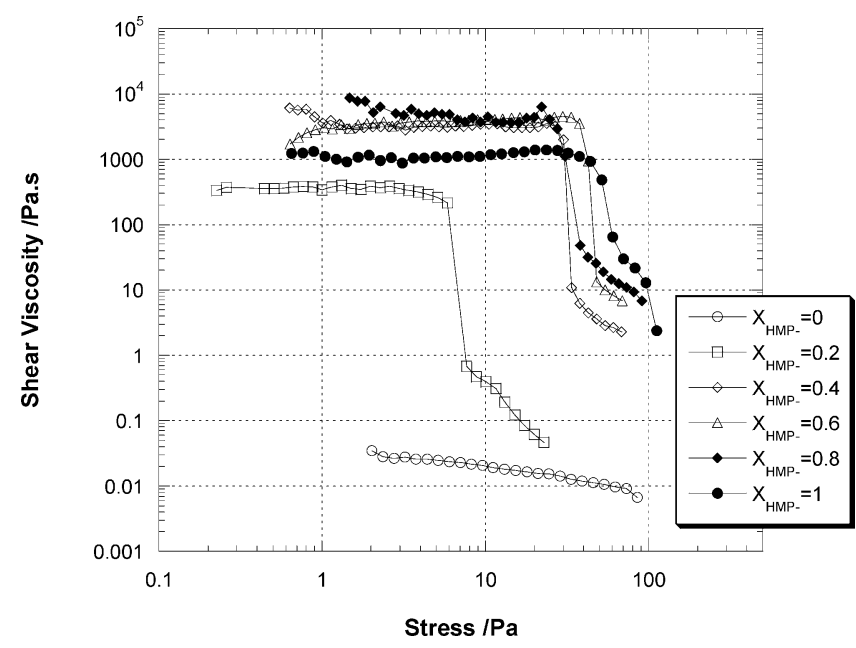

Figure 3. Dependence of shear viscosity $(\eta)$ on the applied stress $(\sigma)$ for different weight fractions, at a total polymer concentration of $1 \mathrm{wt} \%$.

different polymer ratios were investigated, for clarity only the results for three representative samples are displayed. In a solution of only $\mathrm{HMP}^{+}\left(X_{\mathrm{HMP}^{-}}=0\right), G^{\prime \prime}$ (loss modulus) is higher than $G^{\prime}$ (storage modulus) in almost the entire frequency range. $G^{\prime}$ only exceeds $G^{\prime \prime}$ at high frequencies. This indicates that the sample has a viscous behavior at low frequencies and an elastic response at high frequencies. For this sample the intersection between $G^{\prime}$ and $G^{\prime \prime}$ $-f^{*}=1 /\left(2 \pi \tau^{*}\right), f^{*}$ being the intersection frequency and $\tau^{*}$ the longest relaxation time, occurs at high frequencies, indicating short relaxation times. For samples with $X_{\mathrm{HMP}^{-}}$ $=0.5$ and $X_{\mathrm{HMP}^{-}}=1$, it is observed that in the entire frequency range studied, $G^{\prime}$ is higher than $G^{\prime \prime}$. This suggests a dominating elastic response. However, both moduli are considerably higher with $X_{\mathrm{HMP}^{-}}=0.5$ than with $X_{\mathrm{HMP}^{-}}=1$ suggesting that there is a strengthened network at intermediate $X_{\mathrm{HMP}^{-}}$values. The intersection occurs at very low frequencies, indicating quite long relaxation times. Relaxation times were taken directly from the frequency dependence plots. We believe that the crossing between $G^{\prime}$ and $G^{\prime \prime}$ corresponds to the longest relaxation time and that more relaxation times are present. The polymer polydispersity and the polymer reptation within polymer aggregates are examples of other possible processes.

Figure $2 \mathrm{~b}$ illustrates the dependence of complex viscosity on frequency and Figure 3 the dependence of shear viscosity on stress. Analyzing the dependence of complex viscosity on frequency, we can infer an increase of viscoelastic characteristics (higher frequency dependence) at intermediate values of $X_{\mathrm{HMP}^{-}}$. This result is typical for polymer network systems containing cross-links or entanglements. For solutions of $\mathrm{HMP}^{+}$alone $\left(X_{\mathrm{HMP}^{-}}=0\right)$, the complex viscosity is only slightly dependent on frequency, which indicates a considerable number of unentangled polymers. The dependence of complex viscosity on frequency can be described by a power law $\eta^{*}$ $=k f^{-n}$, where $n$ is a measure of the viscoelasticity response of the system. Table 3 shows that higher values of $n$ (indicating a more elastic response) appear at intermediate compositions. Figure 3 shows that all samples display a Newtonian plateau at low stress, indicating that shear viscosity is independent of shear rate or stress in this region. The Newtonian viscosity is higher at intermediate $X_{\mathrm{HMP}^{-}}{ }^{-}$alues. A shear thinning behavior indicates network breakdown. It occurs mainly above $30 \mathrm{~Pa}$. Only less viscous samples have critical stresses below $10 \mathrm{~Pa}$ (stress above which the system starts to be shear-thinning). If the shear viscosity is plotted against the shear rate, it is possible to compare with the complex viscosity plotted against angular frequency. A complex viscosity plateau at low frequencies was not reached. Thus, comparison between complex viscosity and shear viscosity can only be done in the "thinning" curve. It was found that the Cox-Merz rule gives an acceptable relation between the two viscosities. Relaxation times depend on the composition of the mixtures. They display the same trend as the storage modulus and viscosity; i.e., the highest values are at intermediate values of $X_{\mathrm{HMP}^{-}}$. It shows that the dynamics are very slow within this region, due to an increase of association strength, which favors clustering and entanglement. A change of time of intersection with composition is illustrated in Table 2 .

Figure 4 summarizes the dependence of the two rheological parameters, $G^{\prime}$ and complex viscosity $\left(\eta^{*}\right)$, on the $\mathrm{HMP}^{-}$weight fraction in mixtures with a total polymer concentration of $1 \mathrm{wt} \%$. The considerably higher viscosity and storage modulus of $\mathrm{HMP}^{-}$in water than $\mathrm{HMP}^{+}$is in line with the higher molecular mass and the longer persistence length of the polyanion, but we also note that HMCMC is more amphiphilic; thus there are strong contributions to rheology both from entanglements and from hydrophobic association.

It can also be inferred from Figure 4 that mixtures between the two oppositely charged polyelectrolytes give higher viscosities than those observed for the two polymers separately. In line with expectation, mixing of $\mathrm{HMP}^{-}$with a solution of $\mathrm{HMP}^{+}$gives a larger relative change than the opposite. Viscosity and storage modulus display maxima for intermediate mixing ratios. Strong gels are formed at these compositions, with strong interpolymer cross-links. The criterium used to identify strong gels consists of the weak dependence of $G^{\prime}$ on frequency. The maxima occur around a polyanion weight fraction of 0.6 . We note that with dominating electrostatic association the maximum would be expected at the charge neutrality, which corresponds to a value of $\mathrm{HMP}^{-}$of 0.056 , while interpolymer hydrophobic association would be maximal for a matching concentration of hydrophobic substituents, i.e., for $\mathrm{HMP}^{-}=0.80$. The observed maximum corresponds to a combination of electrostatic and hydrophobic associations. Contributions to the network are from electrostatic and hydrophobic associations; charge neutrality is located in the $\mathrm{HMP}^{+}$dominating region and the hydrophobic group 1:1 stoichiometry is located in the $\mathrm{HMP}^{-}$dominating region. The network nature of pure and mixed systems is different. A study regarding homogeneous (expected in pure systems) and heterogeneous polyelectrolyte networks indicates that the heterogeneous network is built by bundles of close-packed polycation and polyanion chains and the effective size of the network is bigger. ${ }^{19}$

Controlling the Electrostatic Association by Adding Salt and Varying the pH. Figures 5 and 6 show the effect of adding salt on the rheology. In Figure 5 is illustrated the influence of salt on the viscoelastic properties for $X_{\mathrm{HMP}^{-}}=0.6$, i.e., close to the maximum in network strength (cf. Figure 4). The storage modulus and the relaxation time decrease with salt addition, indicating a lower number of cross-links and shorter cross-link lifetimes.

Figure 6 shows the dependence of storage modulus $\left(G^{\prime}\right)$ of salt addition for several mixing conditions. The presence of $100 \mathrm{mM}$ of $\mathrm{NaCl}$ induces three different effects: a phase separation at low $X_{\mathrm{HMP}^{-}}$values, a decrease in the viscosity at intermediate compositions, and an increase in the viscosity at high $X_{\mathrm{HMP}^{-}}$. 


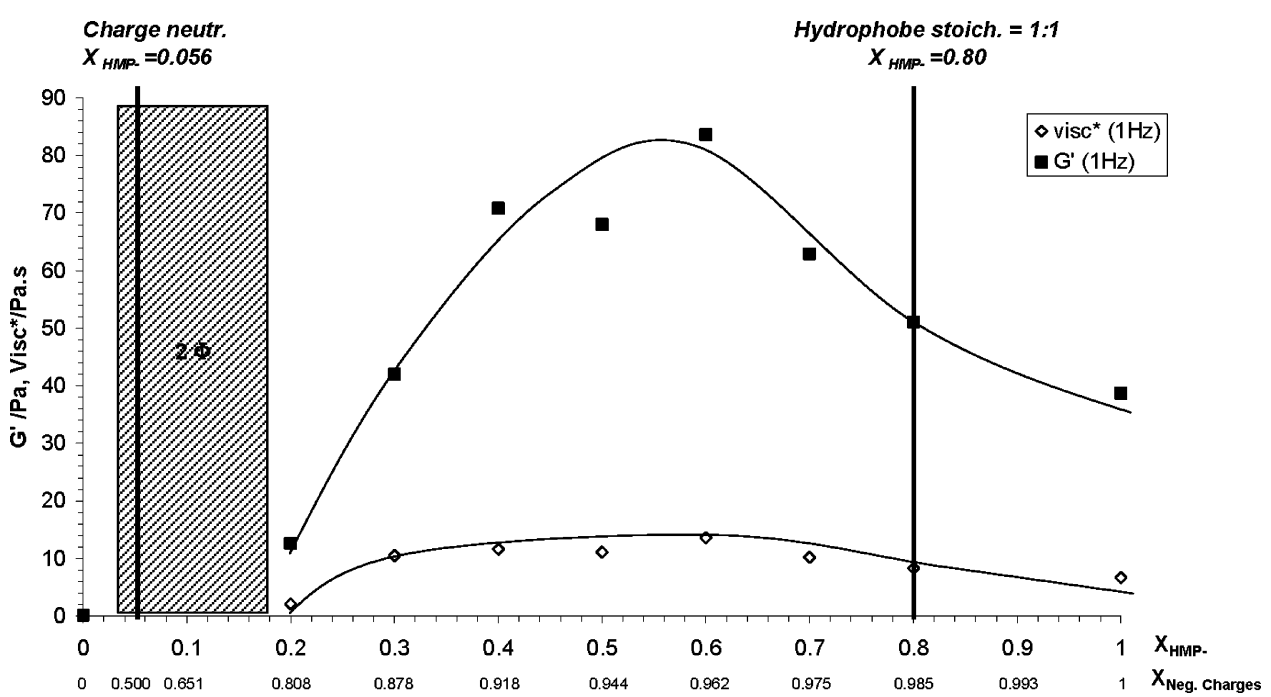

Figure 4. Storage modulus $\left(G^{\prime}\right)$ and complex viscosity (visc*) at $1 \mathrm{~Hz}$ vs $\mathrm{HMP}^{-}$weight and charge fraction, at $1 \mathrm{wt} \%$ total polymer concentration $\left(\mathrm{HMP}^{+}\right.$and $\left.\mathrm{HMP}^{-}\right)$. Charge neutralization and hydrophobes 1:1 stoichiometry are indicated in the figure to simplify the observation. Dotted area indicates phase separation. The lines are a guide for the eyes.

Table 2. Dependence of Relaxation Time $(\tau)$ on $X_{\mathrm{HMP}}$ and on $\mathrm{pH}\left(\right.$ for $X_{\mathrm{HMP}^{-}}=1$ ), at a Total Polymer Concentration of 0.5 wt \%

\begin{tabular}{cccc}
\hline$X_{\mathrm{HMP}^{-}}$ & $\tau / \mathrm{s}$ & $X_{\mathrm{HMP}^{-}}$ & $\tau / \mathrm{s}$ \\
\hline 0 & 0.0265 & 0.6 & 159 \\
0.4 & 79.6 & 0.8 & 106 \\
\hline $\mathrm{pH}\left(X_{\mathrm{HMP}^{-}}=1\right)$ & $\tau / \mathrm{s}$ & $\mathrm{pH}\left(X_{\mathrm{HMP}^{-}}=1\right)$ & $\tau / \mathrm{s}$ \\
\hline 8 & 46.8 & 4 & $>168$ \\
7.2 & 159 & &
\end{tabular}

Table 3. Dependence of $n$ Values (extracted from the power law model, and indicating the viscoelastic responses) on Composition (to a 1 wt \% Total Polymer Concentration) and on pH (to a 1 wt \% Polymer Concentration and $X_{\mathrm{HMP}^{-}}=1$ )

\begin{tabular}{cccc}
\hline$X_{\mathrm{HMP}^{-}}$ & $n$ & $X_{\mathrm{HMP}^{-}}$ & $n$ \\
\hline 0.2 & 0.79 & 0.8 & 0.84 \\
0.4 & 0.84 & 1 & 0.76 \\
0.6 & 0.85 & & \\
\hline $\mathrm{pH}$ & $n$ & $\mathrm{pH}$ & $n$ \\
\hline 8 & 0.66 & 4 & 0.88 \\
7.2 & 0.74 & &
\end{tabular}

In the $\mathrm{HMP}^{+}-$water binary system, small additions of salt $(20 \mathrm{mM})$ decrease slightly the storage modulus (the same trend for viscosity). Further addition of salt (100 $\mathrm{mM}$ ) induces phase separation due to polyelectrolyte insolubility (cf. above). Both these effects can be related to a contraction of the polymer chains on addition of salt. Studies with the used $\mathrm{HMP}^{+}$show that the polyelectrolyte tends to contract in the presence of salt. ${ }^{26}$ The same mechanism applies to the mixed polymer solutions that are dominated by $\mathrm{HMP}^{+}$. Therefore, salt addition can give a phase separation or a decrease in viscosity, depending on conditions. Table 1 also includes the relative viscosity of the top phases in such systems with salt. The viscosity is around three times that of water, and the bottom phases occupy approximately $40 \%$ (v/v). $\mathrm{HMP}^{+}$-rich samples owe their solubility to the dissociating counterions, and therefore, solubility decreases on salt addition. The top phase is depleted with respect to both polymers giving a low viscosity.

When the weight fraction $X_{\mathrm{HMP}^{-}}$is between 0.2 and 0.6 , the storage modulus decreases on salt addition. This is attributed to a weakened electrostatic association between

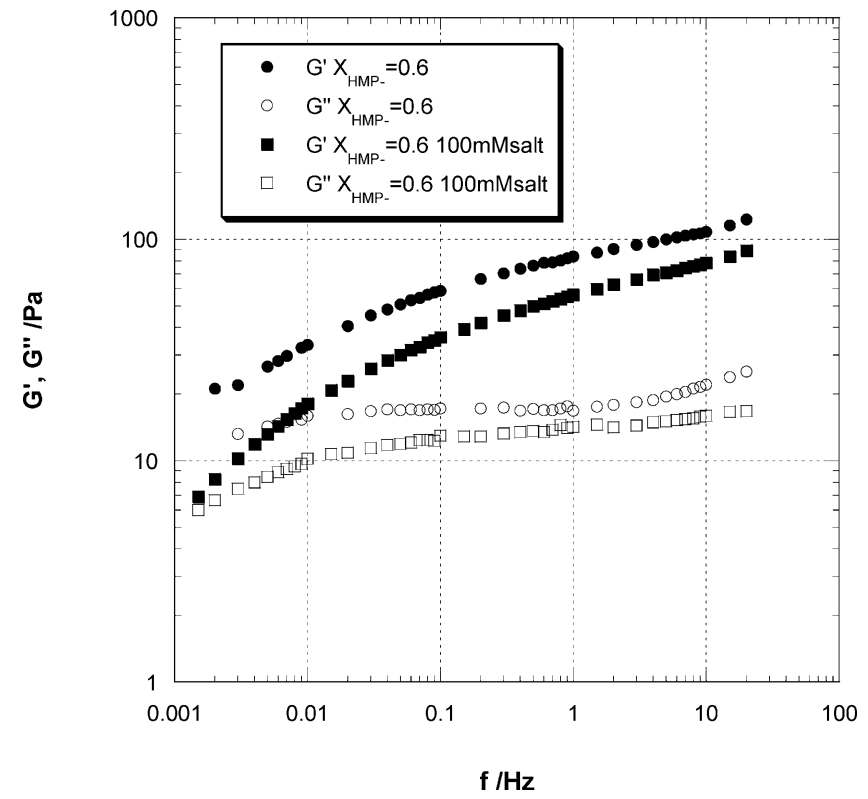

Figure 5. Salt effect on storage modulus $\left(G^{\prime}\right)$ and loss modulus $\left(G^{\prime \prime}\right)$ at different frequencies, for $\mathrm{HMP}^{-}$polymer weight fraction of 0.6 . The total polymer concentration is $1 \mathrm{wt} \%$.

the two polyions. Also, the polycation tends to contract with salt, as argued before, reducing its ability to associate. Within the range of $X_{\mathrm{HMP}^{-}}$values of $0.8-1$, the storage modulus and the viscosity increase in the presence of salt. In this region there is a large excess of negative charges since HMP $^{-}$is in excess and is much more highly charged than $\mathrm{HMP}^{+}$. Without salt, in this range, repulsion between polyanion chains limits the degree of hydrophobic association as approach of polyion chains leads to an unfavorable counterion accumulation. Addition of electrolyte reduces the repulsion and facilitates a threedimensional network development, here due mainly to hydrophobic interactions.

Figure 7 shows the storage modulus and the complex viscosity as functions of $\mathrm{HMP}^{-}$weight fraction, for a total polymer concentration of $0.5 \mathrm{wt} \%$-with and without added salt $(100 \mathrm{mM})$. The results follow the same trend as with $1 \mathrm{wt} \%$, and the interpretation is assumed to be the same. 


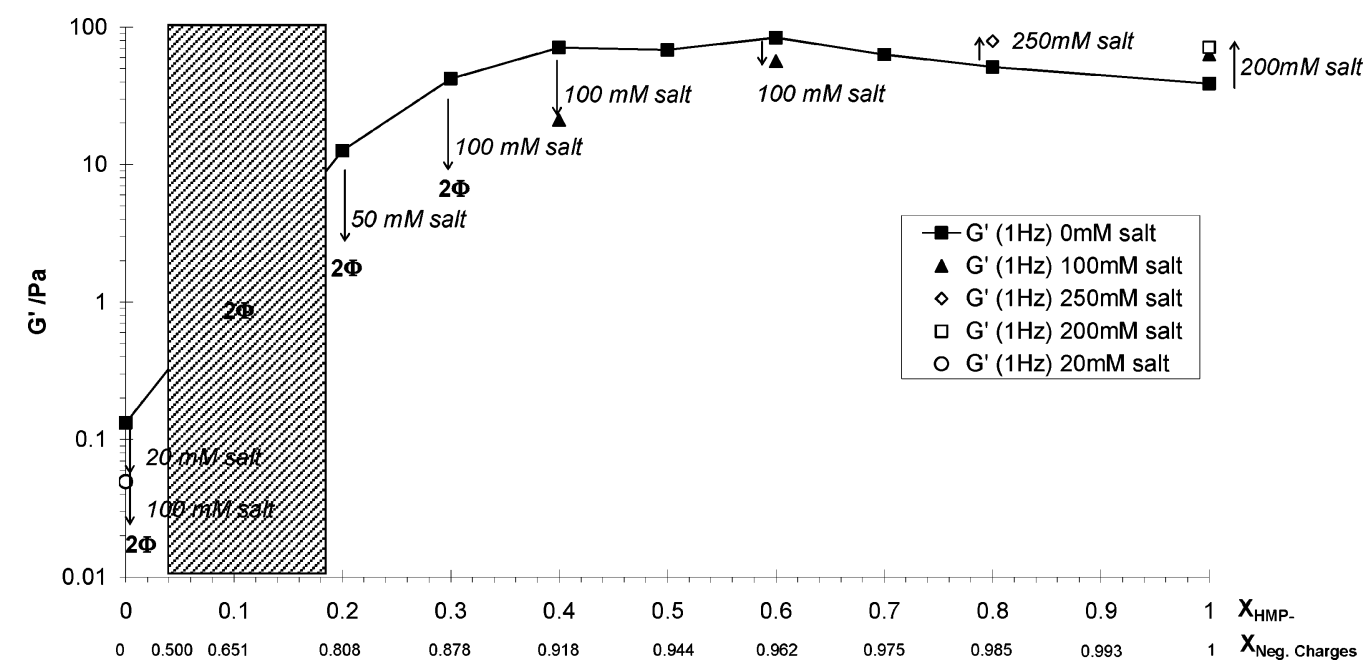

Figure 6. Salt effect on storage modulus $\left(G^{\prime}\right)$ for different $X_{\mathrm{HMP}^{-}}$at $1 \mathrm{wt} \%$ total polymer concentration $\left(\mathrm{HMP}^{+} \mathrm{HMP}{ }^{-}\right)$. Arrows indicate viscosity direction with salt addition; salt concentration is also indicated. Dotted area indicates phase separation. The lines are a guide for the eyes.

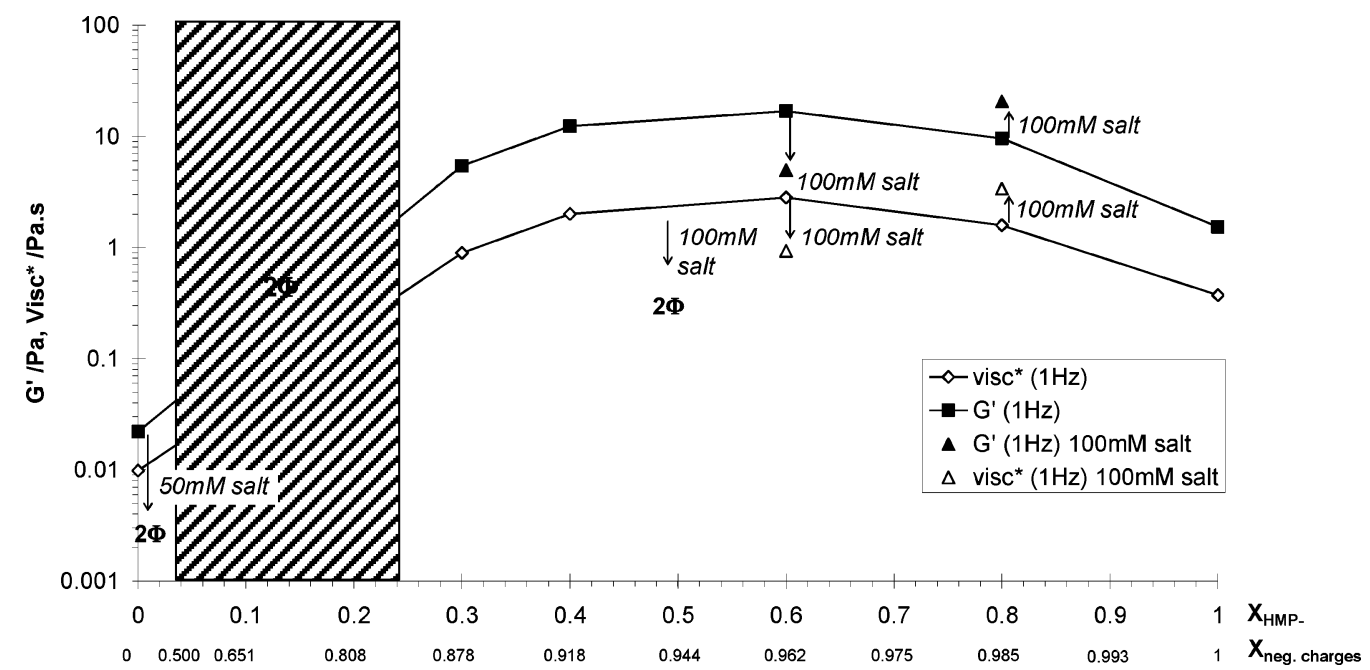

Figure 7. Storage modulus $\left(G^{\prime}\right)$ and complex viscosity (visc*) as a function of $\mathrm{HMP}^{-}$weight fraction. Total polymer concentration is kept at $0.5 \mathrm{wt} \%\left(\mathrm{HMP}^{+} \mathrm{HMP}^{-}\right)$. Arrows indicate viscosity direction with salt addition and are accompanied by the salt concentration. Dotted area corresponds to phase separation region. The lines are a guide for the eyes.

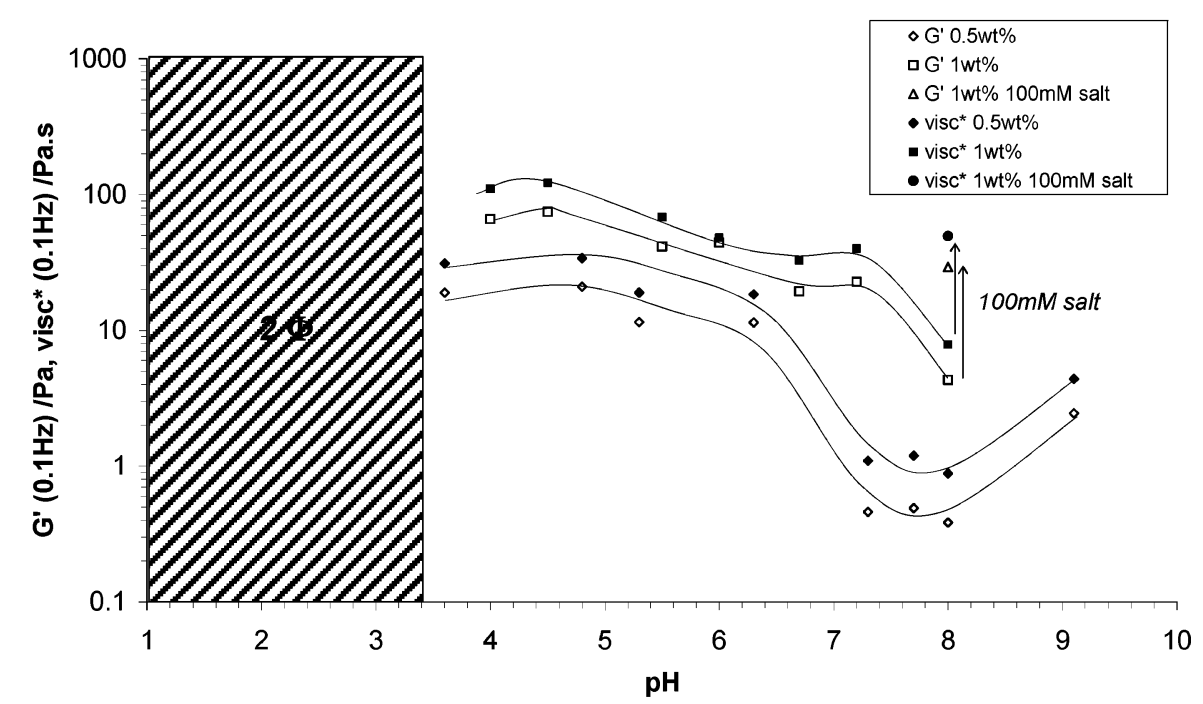

Figure 8. Storage modulus $\left(G^{\prime}\right)$ and complex viscosity (visc*) at $0.1 \mathrm{~Hz}$ as a function of pH, for 1 wt $\%$ of $X_{\mathrm{HMP}^{-}}=1$. Dotted area indicates phase separation region. The lines are a guide for the eyes.

While $\mathrm{HMP}^{-}$is soluble even at quite high ionic strengths, it is not soluble at low $\mathrm{pH}$, where the carboxylate groups are protonated and the charge density decreases; at low enough $\mathrm{pH}$ the polymer has a nonionic character. Studies as a function of $\mathrm{pH}$ regarded two different systems: First, we investigated how $\mathrm{HMP}^{-}$behaves alone 


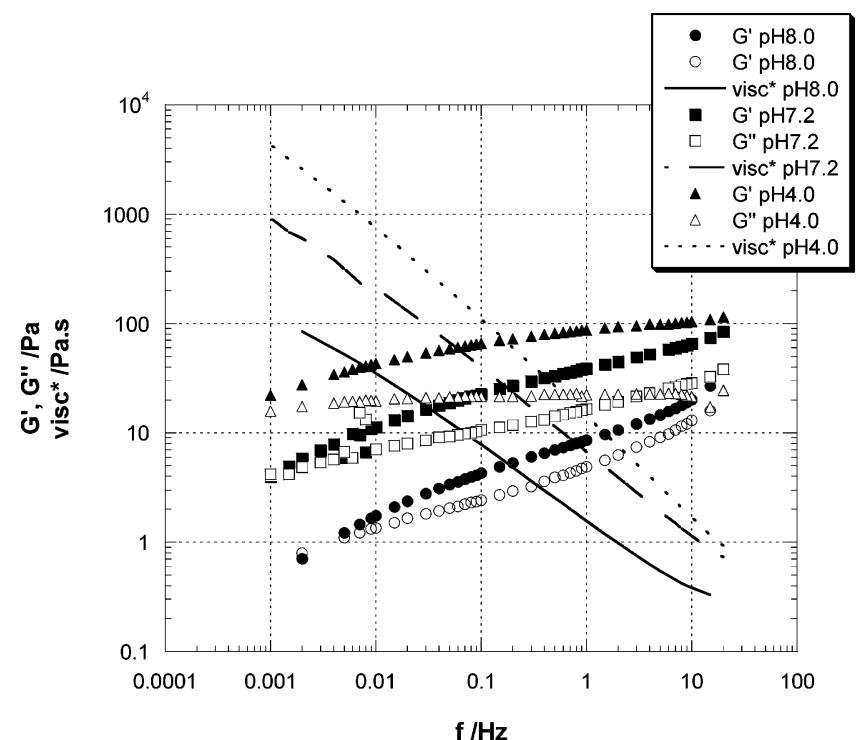

Figure 9. Dependence of the storage modulus $\left(G^{\prime}\right)$, loss modulus $\left(G^{\prime \prime}\right)$ and complex viscosity (visc*) on deformation frequencies, at different $\mathrm{pH}$ values, for $1 \mathrm{wt} \%$ of $X_{\mathrm{HMP}^{-}}=1$.

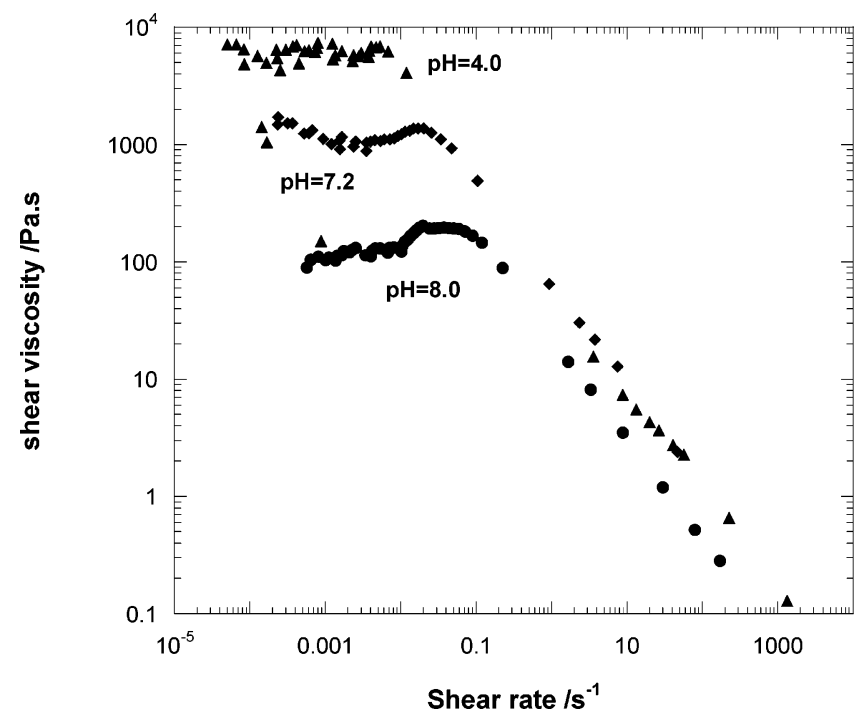

Figure 10. Dependence of shear viscosity with shear rate, at different $\mathrm{pH}$ for $X_{\mathrm{HMP}^{-}}=1$, at a total polymer concentration of 1 wt $\%$. in water (at 0.5 and $1 \mathrm{wt} \%$ ). Second, the mixture system of the two polyelectrolytes was investigated, both with a stoichiometry of $1: 1$ by weight and $1: 1$ by hydrophobes. $\mathrm{HMP}^{-}$dissolved in water gives a $\mathrm{pH}$ of 7.17 at $1 \mathrm{wt} \%$ and of 7.70 at 0.5 wt $\%$.

Figures 8-10 describe rheological responses of the $\mathrm{HMP}^{-}$system (without $\mathrm{HMP}^{+}$added). Figure 8 shows how the storage modulus and complex viscosity at $0.1 \mathrm{~Hz}$ change as a function of $\mathrm{pH}$. It can be observed that the rheological parameters change in the same way with $\mathrm{pH}$, at both 1 and $0.5 \mathrm{wt} \%$ of total polymer concentration. By decreasing the $\mathrm{pH}$, the response becomes more elastic, and the relaxation times longer (Figure 9), with a pronounced jump in the range $\mathrm{pH} 6-8$. The variation of selected relaxation times with $\mathrm{pH}$ is reported in Table 2. A clear maximum in viscosity and storage modulus was detected around $\mathrm{pH}$ 5. Thus, more and more long-lived cross-links are formed at this $\mathrm{pH}$. At $\mathrm{pH}$ below 5 a decrease of viscosity and storage modulus is found in the system. At a $\mathrm{pH}$ below 3.5, the polymer solution shows a biphasic region, where a dilute phase coexists with $\mathrm{HMP}^{-}$(now less soluble due to the virtual absence of charges). The nonmonotonic dependence of viscosity on $\mathrm{pH}$ can be explained with structural aspects in $\mathrm{HMP}^{-}$. Lowering the polymer charge density on protonation leads at first to a decreased electrostatic repulsion and thus a stronger network. The lower viscosity at lower charge densities is interpreted in terms of an extensive association between polymer chains, approaching local phase separation, eliminating the possibility of forming an extensive threedimensional network; at slightly lower charge densities macroscopic phase separation follows. This picture is in line with work by Cohen Stuart and co-workers, who observed that the apparent hydrodynamic radius of $\mathrm{HMP}^{-}$ has a maximum at $\mathrm{pH} 5$. This maximum can be interpreted in terms of an interplay between the electrostatic expansion of the polymer chains due to charges and aggregate formation. At lower $\mathrm{pH}$ values the polymer chains are less swelled but more aggregated, while above $\mathrm{pH} 5$ electrostatic repulsion becomes dominating and the aggregates break up. ${ }^{24}$

Figures 9 and 10 illustrate the rheological behavior for selected $\mathrm{pH}$ values. Figure 9 shows how the storage and loss moduli, as well as the complex viscosity, change with frequency, for different $\mathrm{pH}$ values. As can also be seen the relaxation times are longer at lower $\mathrm{pH}$. Table 3 indicates also the $n$ values extracted from the power law model. On

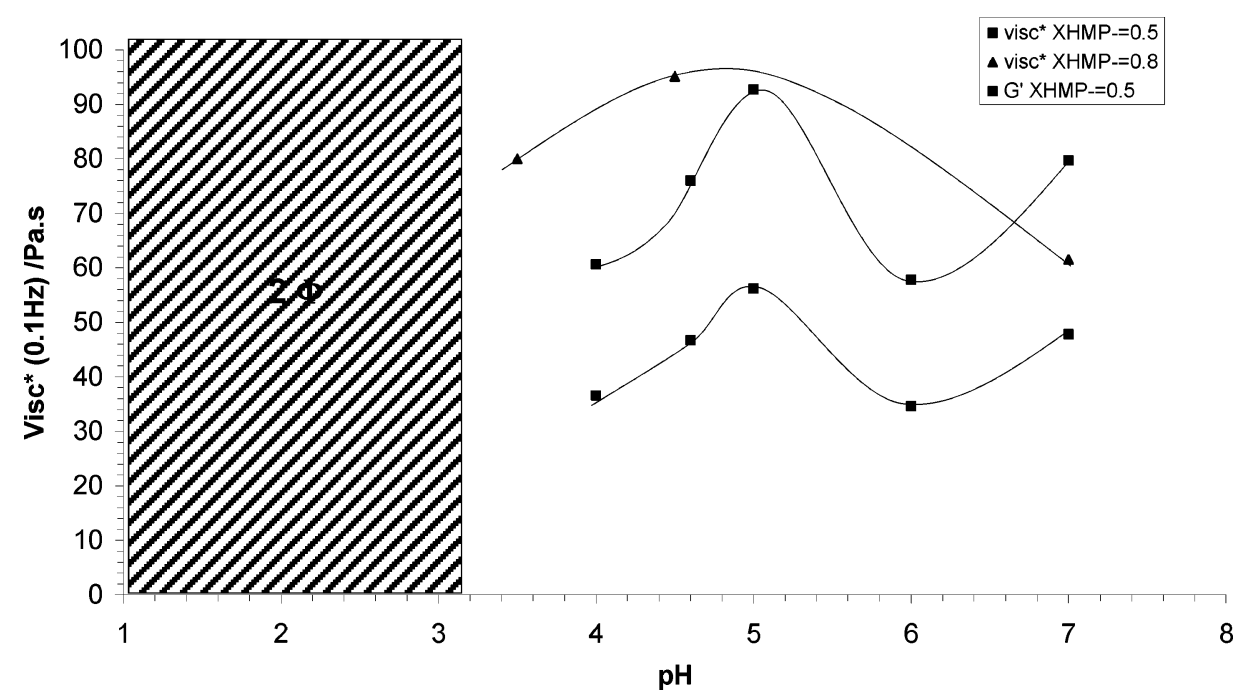

Figure 11. Complex viscosity (visc*) at $0.1 \mathrm{~Hz}$ as a function of $\mathrm{pH}$, for a stoichiometry $1: 1$ by weight $\left(X_{\mathrm{HMP}^{-}}=0.5\right)$ and $1: 1$ by hydrophobes $\left(X_{\mathrm{HMP}^{-}}=0.8\right)$ for a total polymer concentration of $1 \mathrm{wt} \%$. The lines are a guide for the eyes. 
increase of the $\mathrm{pH}$, a decrease in $n$ values (and thus, a lower solidlike character) is seen. Figure 10 displays the shear tests, where the Newtonian plateau is found to increase with a $\mathrm{pH}$ decrease (down to $\mathrm{pH} 4$ ), indicating more cross-links and higher relaxation times at this $\mathrm{pH}$. Again, this can be explained by a decrease of the interpolymer repulsion by decreasing the $\mathrm{pH}$, and thus, promotion of aggregation. The $\mathrm{COO}^{-}$groups are being progressively converted to $\mathrm{COOH}$ groups and the reduced repulsion between polymer chains facilitates interpolymer hydrophobic association. Another observation, which agrees with this explanation, is the increase of viscosity when salt is added to a polymer solution of $\mathrm{pH}$ 8. At a $\mathrm{pH}$ around $2, \mathrm{HMP}^{-}$becomes essentially nonionic giving a lowered solubility and phase separation. The persistence length is also influenced by the degree of protonation. With decrease of the $\mathrm{pH}$ a less stiff polymer with lower persistence length would be expected and thus a decrease in viscosity. However, the polymer flexibility probably plays a minor role compared to the decrease in repulsion between the $\mathrm{HMP}^{-}$chains.

The effect of addition of $\mathrm{HMP}^{+}$at different $\mathrm{pH}$ values is shown in Figure 11. It shows the $\mathrm{pH}$ dependence of the complex viscosity and storage modulus for different mixing ratios of $\mathrm{HMP}^{+}$and $\mathrm{HMP}^{-} . X_{\mathrm{HMP}^{-}}$was locked at 0.5 (1:1 by weight) and at 0.8 (1:1 by hydrophobes). The storage modulus and the viscosity have a maximum when $\mathrm{pH}$ is around 5 for all cases. An interesting minimum around pH 6 is seen; further studies are needed to identify the underlying mechanism. Due to the similarity, we believe that $\mathrm{HMP}^{-}$conformation is responsible for the detected results, as explained in relation to Figure 8 . Below $\mathrm{pH} 3$, the mixture phase separates; this is referred to charge neutralization. At $\mathrm{pH} 3-5$, weak aggregates form. Above $\mathrm{pH} 5$, the fraction of negative charges is high, which contributes to weaker attractive forces and thus to a decrease of network strength. This is seen by a further decrease in viscosity.

\section{Conclusions}

Hydrophobic modification of oppositely charged polymers prevents, to a large extent, the typical associative phase separation detected for the polyelectrolytes in general. This can be understood in terms of a combination of electrostatic and hydrophobic association, the latter stabilizing aggregates with a net charge. Viscosity and storage modulus are significantly higher in the mixture of the two HM polyelectrolytes compared to each polymer alone in solution. The strongest network is formed in the weight fraction where two different effects are superposed: electrostatic and hydrophobic. For intermediate concentrations (where both electrostatic and hydrophobic effects are significant), a strong interconnected heterogeneous network is established, as seen by an increase of viscosity, relaxation times, and storage modulus.

Viscosity can be controlled by salt addition. In the HMPdominating region, salt decreases electrostatic repulsion between the long $\mathrm{HMP}^{-}$chains and increases the viscosity. At intermediate mixture ratios, viscosity is decreased by salt due to $\mathrm{HMP}^{+}$contraction and a decrease of the number of cross-links.

Charge density of $\mathrm{HMP}^{-}$(and therefore, the association with $\mathrm{HMP}^{+}$) can be controlled by $\mathrm{pH}$. Down to $\mathrm{pH} 5, \mathrm{HMP}^{-}$ becomes less charged and, thus, repulsion between $\mathrm{HMP}^{-}$ chains is reduced, contributing to a large number of crosslinks and thus to a higher viscosity. A further decrease in $\mathrm{pH}$ results in a decrease of viscosity, explained by an extensive close association on approach of the phase separation point. This applies both to the binary $\mathrm{HMP}^{-}$ aqueous solutions and to the ternary $\mathrm{HMP}^{-}-\mathrm{HMP}^{+}$ solutions.

Acknowledgment. F.E.A. acknowledges Fundação para Ciência e Tecnologia (FCT) for a research grant (ref. SFRH/BD/8168/2002). The Colloid Group in Coimbra University is supported by grants from the Fundação para Ciência e Tecnologia (ref. POCTI/QUI/35415/99 and POCTI/QUI/45344/02). Dr. Leif Karlson from Akzo Nobel Surface Chemistry is acknowledged for kindly supplying the hydrophobically modified polymers. We thank Dr. Krister Thuresson and Dr. Cesare Oliviero for fruitful comments.

LA050590K 